
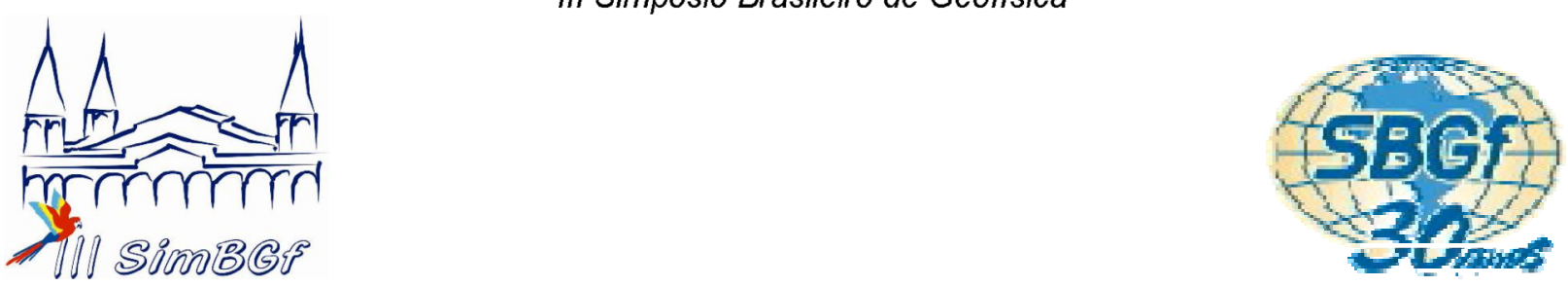

\title{
Indícios de mineralização em área contaminada por derivados de petróleo.
}

récar Aııııcto Mnreira Antônin Celan de Oliveira Rrana I ennardn 7ani Cactelln Menartamento de Fenlnnia Anlicada, IGCE/UNESP - Campus Rio Claro.

Copyright 2008, SBGf - Sociedade Brasileira de Geofisica

Este texto foi preparado para a apresentação no III Simpósio Brasileiro de Geofísica, Belém, 26 a 28 de novembro de 2008 . Seu conteúdo foi revisado pelo Comitê Técnico do III SimBG nos ño associados. E prob ha necessañ

\section{Resumo}

Estudos geofísicos em áreas impactadas por derivados de petróleo descrevem anomalias tanto de baixa resistividade quanto de elevada resistividade, confirmada como fase contaminante por meio de análises químicas. Este aparente contradição pode refletir processos de degradação, associado ao tempo de residência de contaminantes no meio, por meio da geração de subprodutos que alteram as propriedades físicas do meio, principalmente pela dissolução mineral por ação de ácidos orgânicos e pela neoformação de minerais de óxidos e hidróxidos de ferro. Este trabalho apresenta resultados da aplicação do método de eletrorresistividade e polarização induzida, em uma área industrial

dicloroetano e sais inorgânicos. Sob a ótica dos processos de atenuação natural, são discutidos indícios de mineralização na porção central e adjacências da área contaminada.

\section{Introdução}

Os métodos geofísicos são uma alternativa no diagnóstico de áreas contaminadas, devido à rapidez e o baixo custo em estudos ambientais. Esta ferramenta pode auxiliar na detecção e no monitoramento de áreas contaminadas, em conjunto com métodos diretos de investigação, como poços de monitoramento. As técnicas geofísicas de Sondagem Elétrica Vertical (SEV). Radar de Penetração no Solo (GPR) e Eletromagnético Indutivo (EM) são aplicáveis na caracterização e monitoramento de áreas impactadas por contaminantes por Fase Líquida Não Aquosa (NAPL) (Atekwana et al., 2000; Redman et al., 1994)

Diversos trabalhos descrevem a aplicação dos métodos geofísicos combinados com análises diretas de investigação do meio impactado, na caracterização de contaminantes derivados de petróleo em diversas áreas.

A aplicação combinada da técnica de Caminhamento Flótricn a Snndanem Flétrica Vertical em lima et al. (1995), descrevem a detecção de plumas de contaminação provenientes de um aterro de resíduos líquidos derivados de petróleo, cuja correlação entre pseudo-seções de resistividade aparente e um número muito reduzido de poços de monitoramento permitiu associar intervalos de baixa resistividade com áreas de concentração de contaminantes.

A resposta geofísica em áreas contaminadas por derivados de hidrocarbonetos apresenta anomalias de alta e de baixa resistividade. Esta aparente incongruência pode ser esclarecida por meio da correlação do tempo de residência dos contaminantes no solo e tempo de exposição a processos de degradação.

Alguns trabalhos sugerem que a biodegradação de compostos (LNAPL) pode mudar as propriedades geoquímicas do aqüífero de forma acentuada que podem chegar a alterações significativas em medidas de resistividade e na propagação de ondas eletromagnéticas (Sauck, 2000; Atekwana et al., 2000). Estudos em escala de laboratório sugerem que a biodegradação de LNAPL sob condições aeróbicas e anaeróbicas podem mudar de forma substancial as condições biogeoquímicas do aqüífero impactado, resultando em reduções drásticas de resistividade elétrica (Cassidy, et al., 2001)

A degradação de contaminantes por ação de microorganismos, denominada biodegradação, é um dos principais mecanismos responsáveis pela degradação de contaminantes com longos períodos de residência no ambiente. A eficiência deste processo depende de uma conjunção de fatores, como a presença de receptores elétricos (O2, SO42-, $\mathrm{Fe} 3+, \mathrm{Mn} 4+, \mathrm{NO} 3-)$, condições adequadas de $\mathrm{pH}, \mathrm{Eh}$, temperatura e baixo teor de contaminação (EPA, 1998).

Este processo resulta na mineralização dos elementos em solução. Diversos trabalhos descrevem mineralizações de hematita $\left(\mathrm{F} \in \mathrm{O}_{3}\right)$ e Magnetita $(\mathrm{FeO})$ em áreas contaminadas (Lovley, 1990; McCabe et al, 1987), seja em locais sob estritas condições anaeróbicas ou em áreas sob condições anaeróbicas. Desta forma, a presença de neoformações minerais contribui para redução nos valores de resistividade e aumento nos valores de cargabilidade.

Este trabalho apresenta os resultados de aplicação dos métodos de eletrorresistividade e polarização induzida, em uma área industrial contaminada por benzeno, tolueno, xileno, 1,2 dicloroetano e sais inorgânicos. São discutidas as alterações físicas do meio contaminado por meio dos resultados obtidos e na perspectiva dos processos de biodegradação de compostos NAPL, além de discutir indícios de mineralização na porção central e adjacências da área contaminada. 


\section{Atenuação Natural}

A Atenuação Natural compreende um trabalho de remediação que inclui uma variedade de processos físicos, químicos e biológicos que, dentro de condições favoráveis, permite a redução de massa, toxicidade, mobilidade, volume ou concentrações de contaminantes no solo ou água subterrânea sem intervenção humana (EPA 1999). Este processo ocorre naturalmente em qualquer área contaminada, com variável grau de eficiência, dependendo dos tipos e concentrações de contaminantes presentes e dos processos físicos, químicos e biológicos característicos do solo e da água subterrânea (EPA, 1999)

Este processo é importante para a degradação de derivados de petróleo, que podem ser degradados por organismos encontrados em quase todos os ambientes. Vários compostos LNAPLs como os BTEXs, podem ser degradados em condições ambientais (EPA, 1998).

Contudo, este processo natural pode ser limitado pela falta de nutrientes, elétrons receptores ou ambos (Thomas \& Ward, 1989). Em muitos locais, o processo de biodegradação fica limitado às zonas periféricas da área contaminada, pois a presença de contaminante em elevadas concentrações torna 0 ambiente extremamente hostil a proliferação de microorganismos (Wilson \& Jones, 1993; Rabus \& Heider, 1998).

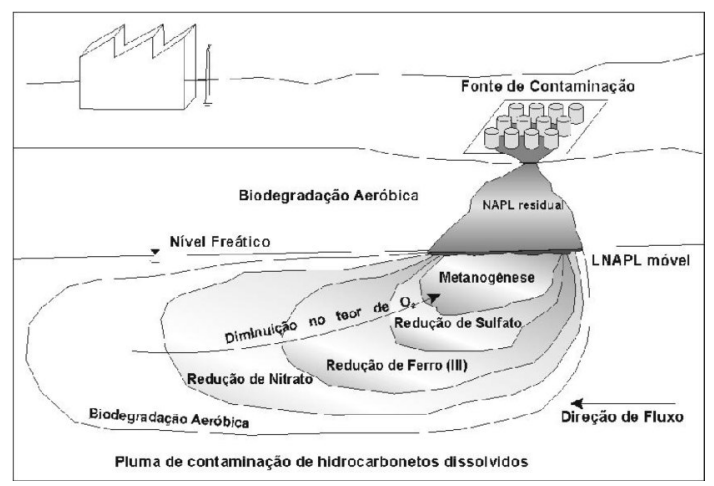

Figura 1 - Zonas de degradação de hidrocarbonetos )

A degradação de derivados de petróleo por metabolismo microbial produz uma variedade de subprodutos reativos, muitos dos quais interagem diretamente com solutos inorgânicos dissolvidos e minerais presentes. Estes subprodutos podem acelerar reações de dissolução e nrec:initac:ãn commlexac:ãn de ínns metálirns em solurãnn, alterações no estado de valência de íons metálicos e mobilização de metais solúveis (Harter, 1977).

Poucos trabalhos abordam a dissolução mineral por ação de ánirnce nrrânionce e cistemas anïífernc racnc, provenientes da degradação de contaminantes derivados de netrólen (Mr.Mahnn at al 1995. Hiehert \& Rennett, 1992).

A dissolução mineral pode desencadear processos como:

- Geração de porosidade intragranular que inibe a recuperação de íons metálicos e derivados de petróleo em aqüíferos de elevada porosidade (Stoessel \& Pittman, 1990).

- Dissolução de óxidos de ferro que pode resultar na liberação de íons metálicos em determinados ambientes (McCabe et al., 1987).

- Neoformação mineral e fechamento de espaços modificação no padrão de circulação da água subterrânea, que dificultam os esforços de remediação.

\section{Localização e Descrição da Área de Estudo}

A área de estudos está localizada no município de

da rodovia SP 191, que liga as cidades de Araras e Rio Claro (Figura 2). O acesso a partir da rodovia SP 330 é feito no cruzamento com a rodovia SP 191, a partir do qual o local de estudos está distante cerca de $1400 \mathrm{~m}$ sentido Rio Claro.

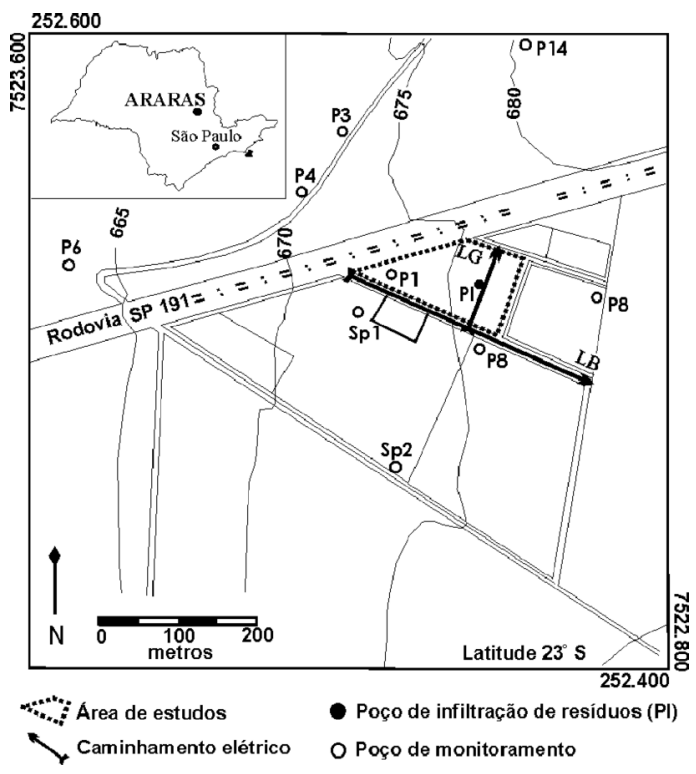

Figura 2 - Localização da área

O local foi ocupado pela Redistil Indústria Química Ltda e pela Indústria Química Sulfabrás S/A, entre os anos de 1981 e 1992. Dentre os produtos fabricados estão o

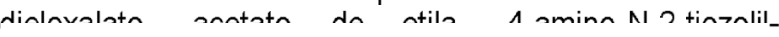
hansana oulfanomidn anntaniriusnta Na matiln 5 matil 2 carboxiamidoxazol (carboxiamido), e utilização de 1,2 dicloroetano e tolueno como solventes (CETESB, 1997).

Os solventes utilizados por ambas as indústrias eram reutilizados após passarem por colunas de destilação. As águas residuais deste processo passavam por um tacho para concentrar os efluentes. Em seguida, os efluentes ceradns eram infiltradns em $\mathrm{um}$ nnco nresente no Incal, procedimento realizado entre 1981 e 1988.

Em novembro de 1985 foram detectados odores em poços de abastecimento nos arredores da área de infiltração. Análises químicas realizadas em amostras coletadas em poços próximos a indústria indicaram 
contaminação da água subterrânea. Posteriormente foram efetuadas análises químicas nos resíduos infiltrados com vistas á caracterização de sua composição química, cujo resultado permitiu correlação com os contaminantes presentes nas águas subterrâneas (CETESB, 1997).

Com o fechamento da indústria a partir de 1992, a nammanhin No Tnannanorin No Cannommanto Amhinntal CETESB, agência ambiental do estado de São Paulo, foi iniciado o monitoramento da atenuação natural dos contaminantes por meio de diversos poços de monitoramento instalados na área e adjacências (Tabela 1, em conjunto com a aplicação de técnicas geofísicas (Figura 3) (CETESB, 1997; Aquino 2000, Moreira \& Dourado, 2003).
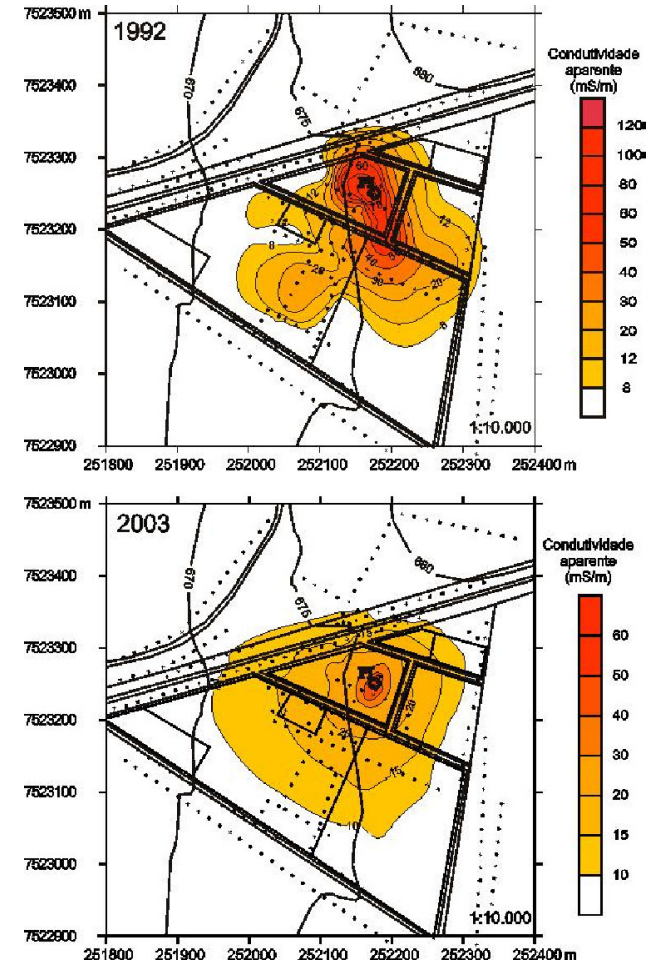

Figura 3 - Mapas de condutividade aparente obtidos em 1992 e 2003, para 7,5m de profundidade (Moreira \& Dourado, 2005)

Tabela 1 - Análises químicas em amostras de água coletadas nos poços de monitoramento em 1992 (CETESB, 1997)

\begin{tabular}{|c|c|c|c|c|c|c|}
\hline Poço & Benzeno* & Tolueno* & Xileno* & 1,2 Dicloroetano * & Cloreto ** & Sulfato** \\
\hline P1 & 543,0 & 117,0 & 10,0 & - & $1,5 \times 10^{3}$ & 30,0 \\
\hline P2 & - & $<1,0$ & - & - & $<0,5$ & 26,0 \\
\hline P3 & - & 31,0 & 21,0 & - & $<0,5$ & 20,0 \\
\hline P4 & 146,0 & - & - & - & 110,0 & 27,0 \\
\hline P5 & 77,0 & & & & 650 & 25,0 \\
\hline PO & 33,0 & $<1,0$ & - & $<1,0$ & 355 & 24,0 \\
\hline$P 7$ & - & - & - & - & 1,0 & 24,0 \\
\hline P8 & $6,0 \times 10^{3}$ & $7,0 \times 10^{3}$ & - & 38,0 & $2.15 \times 10^{3}$ & 31,0 \\
\hline
\end{tabular}

Sucessivas análises químicas realizadas em amostras coletadas no poço de monitoramento P8 indicam decréscimo no teor de contaminantes ao longo de 9 anos (Tabela 2)

Tabela 2 - Análises químicas em amostras coletadas no poço de P8 (CETESB, 1997)

\begin{tabular}{|lccc|}
\hline & $06 / 1990$ & $01 / 1992$ & $11 / 1999$ \\
\hline Benzeno* $^{*} 29,5 \times 10^{3}$ & $6,0 \times 10^{3}$ & $1,19 \times 10^{4}$ \\
Tolueno* $^{*}$ & $24,5 \times 10^{3}$ & $7,0 \times 10^{3}$ & $1,39 \times 10^{4}$ \\
1,2 Dicloroetano* & $12 \times 10^{3}$ & 36,0 & 15,6 \\
Sodio $^{* *}$ & 200,0 & $2,1 \times 10^{3}$ & - \\
Cloreto $^{* *}$ & $2,8 \times 10^{3}$ & $1,8 \times 10^{3}$ & $1,35 \times 10^{3}$ \\
Sulfato** & 32 & 31 & $<10$ \\
\hline - não detectado & * valores em ug/L & $* *$ valores em mg/L \\
\hline
\end{tabular}

\section{Geologia da Área de Estudo}

A geologia local é caracterizada por siltitos argilosos pertencentes à Formação Tatuí, alterados em superfície para solo vermelho escuro argiloso, com matriz de fração silte entre 1 e 3 metros de profundidade e matriz de fração argila entre 3 a 13 metros de profundidade, com transição gradual entre os níveis. Perfis de solo obtidos fora da área contaminada descrevem horizontes lateríticos e a presença de concreções ferruginosas provenientes da migração de ferro em solução para níveis mais profundos do solo.

$\cap$ nível freáticn ectá citıadn entre 107 ค 141 metrnc, com oscilações máximas entre 7,9 e 14,9 metros. O fluxo da água subterrânea nas proximidades dos pontos de infiltração de resíduos é para sudoeste. A condutividade monitoramento varia de $4,4 \times 10^{-7} \quad 7,0 \times 10^{-7} \quad$ de (CETESB 1997, Aquino 2000).

\section{Métodos Utilizados}

O método de Eletrorresistividade é baseado na geração de um campo elétrico em subsuperfície por meio da injeção de corrente elétrica (I), a partir de um dispositivo eletródico, representado por hastes metálicas e

potencial elétrico $(\Delta \mathrm{V})$ produzido pela passagem deste campo e captado por meio de um circuito receptor, que também pode ser representado por hastes metálicas ou eletrodos impolarizáveis. A aplicação da Lei de Ohm considerando o espaçamento entre os eletrodos, representado por um fator denominado $\mathbf{K}$, permite medidas do parâmetro resistividade aparente $(\rho \boldsymbol{a})$ para vários níveis de profundidade (equação 1)

$$
\rho a=\ldots \quad \frac{\Delta V}{I} . \Omega \mathrm{m}
$$

A polarização induzida (IP) é um fenômeno de estimulação de corrente elétrica, observado após o retardo ou atraso de um sinal de voltagem em materiais de subsuperfície (Sumner, 1976). Este método consiste no emprego de um dispositivo eletródico de transmissão 
para injeção de corrente elétrica pulsante e periódica em subsuperfície, cuja resposta é obtida por meio de um circuito receptor constituído de eletrodos impolarizáveis. Os resultados foram obtidos em domínio do tempo, onde a definição de valores medidos em termos da curva da curva transiente indica a presença de materiais polarizáveis em profundidade. A forma mais utilizada de medição no domínio do tempo é em termos de cargabilidade (M), medido em milissegundos (ms) (equação 2):

$$
M=\cdot \frac{1}{V_{c}} \int_{t 2}^{t_{1}} V_{t} d t \quad \mathrm{~ms}
$$

Fste trahalhn anlirnu a técnicra de caminhamentn elẹtricn, em arranjo dipolo-dipolo com espaçamento de $10 \mathrm{~m}$ entre eletrodos e 5 níveis de aquisição de dados em profundidade, totalizando $450 \mathrm{~m}$ dispostos em duas linhas, sendo a linha LG com $120 \mathrm{~m}$ e a linha LB com $330 \mathrm{~m}$ de comprimento (Figura 2). Foram utilizados eletrodos impolarizáveis do tipo Petiau (Petiau, 2000), a hace de Ph-Phr.l2 imerce em caulim $\mathrm{cnm} \mathrm{nH}$ rontroladn, devido a grande estabilidade do eletrólito. Foi utilizado o resistivímetro Terrameter SAS 4000, fabricado pela ABEM instrumentos (Suécia).

Os resultados foram processados no programa Res2dinv (Loke \& Barker, 1996), com aplicação do mesmo tipo de processamento para todas as seções, para possibilitar uma análise da evolução temporal da contaminação.

\section{Resultados}

$\cap$ flı̀n d'ánıla cıluhterrânea senıı centidn sıidnecte, paralelo em sentido contrário à linha LG e cruza de forma ortogonal a linha LB. As sucessivas análises químicas em amostras do poço P8 indicam que o teor de contaminantes foi reduzido drasticamente entre $1990 \mathrm{e}$ 1992, seguido por queda menos acentuada entre 1992 e 1999 (Tabela 3).

Os mapas de condutividade aparente indicam que os maiores valores de condutividade estão associados ao poço de injeção de resíduos, o qual apresenta elevados teores de contaminação (Tabela 1). Esta associação indica que a fase contaminante não está limitada a área da indústria responsável pela contaminação, e que a água subterrânea na indústria vizinhas também está comprometida (Figura 3)

Houve forte redução nos valores de condutividade aparente de 1992 para 2003. Em 1992 os valores máximos de condutividade atingiam $120 \mathrm{mS} / \mathrm{m}$ no poço de injeção de resíduos, enquanto que em 2003 os valores associados ao mesmo ponto atingem o máximo de 60 $\mathrm{mS} / \mathrm{m}$ (Figura 3). Este decréscimo na condutividade é acompanhado por um pequeno aumento na área da anomalia

O poço de injeção de resíduos está localizado na posição $52,5 \mathrm{~m}$ da linha $L G$, abaixo do qual ocorre uma anomalia de alta resistividade no ano de 2004, principalmente entre 6 e $12 \mathrm{~m}$ de profundidade (Figura 4A). Os intervalos laterais apresentam valores de resistividade abaixo dos
$10 \Omega \mathrm{m}$, em contraste bastante acentuado aos valores máximos da parte central, em torno de $150 \Omega \mathrm{m}$.

A anomalia de alta resistividade identificada em 2004 desaparece na seção de 2007, onde predomina uma faixa com valores de resistividade abaixo dos $10 \Omega \mathrm{m}$, seja na porção abaixo do poço de injeção de resíduos ou em suas áreas laterais (Figura 4B). Os valores médios de resistividade são bastante elevados na seção de 2007 em relação à seção de 2004

A linha LG de IP apresenta baixos valores de cargabilidade à montante do fluxo $\mathrm{d}$ água subterrânea, elevada cargabilidade na exata posição da anomalia de resistividade identificada na seção de 2004 , superiores a $36 \mathrm{~ms}$, e valores intermediários a jusante do intervalo resistivo (Figura $4 \mathrm{C}$ ).

A linha LB apresenta um amplo intervalo de baixa resistividade em formato triangular na seção de 2004 -. -.. , . . . . . . m essencialmente limitados a isolinha de condutividade aparente de $20 \mathrm{mS} / \mathrm{m}$ de 2003 (Figura 4). A porção mais rasa deste polígono de baixa resistividade corresponde ao início da linha LG em superfície (Figura 2).

A linha LB de 2007 apresenta intervalos com resistividade abaixo dos $10 \Omega \mathrm{m}$ intercalados por intervalos de elevada resistividade. $O$ intervalo correspondente ao centro de baixa resistividade da seção de 2004 revela na seção de 2007 a presença de uma anomalia de elevada resistividade, com valores superiores a $200 \Omega$ m (Figura $5 B$ ). A faixa de ocorrência desta anomalia indica a presença de um corpo com elevada cargabilidade, com valores acima de $98 \mathrm{~ms}$, segundo a seção de IP da linha LB (Figura 5C).

\section{Discussão e Conclusões}

O desaparecimento da anomalia central de elevada resistividade da linha LG de 2004 na seção de 2007, provavelmente indica a degradação da fase residual de contaminantes restante em solo imediatamente abaixo do poço de injeção de resíduos.

Atingida a solubilidade máxima em água, a fase

partir deste momento, são criadas condições favoráveis para uma ação efetiva do processo de degradação por microorganismos, devido à redução da toxicidade e aumento no teor de oxigênio (Figura 1)

A coincidência de valores de elevada cargabilidade no intervalo da anomalia resistiva de 2004 , indica que além da dissolução mineral em curso na área, demonstrado a

em 2007 onde predominavam valores superiores $150 \Omega . m$ em 2004, também esteja havendo neoformação de minerais. Este intervalo apresenta as menores quantidades de oxigênio disponíveis em subsuperfície na área, por se tratar no centro dispersor de poluentes (Figura 1). Desta forma, os minerais formados neste intervalo são característicos de zonas redutoras.

O amplo intervalo com resistividade inferior a $10 \Omega \mathrm{m}$ na linha LB de 2004 indica a ausência de fase residual em 
solo, com presença somente de fase dissolvida e produtos do processo de degradação dos derivados de petróleo por ação de microorganismos. Esta ausência indica que esta linha está posicionada nas adjacências da área central de injeção de resíduos, conforme demonstrado nos mapas de condutividade aparente.

O intervalo central resistivo da linha LB de 2007, com valores superiores a $200 \Omega \mathrm{m}$ onde anteriormente havia valores inferiores a $10 \Omega \mathrm{m}$, indicam o desaparecimento da fase dissolvida e dos produtos de degradação microbial, conforme indícios sugeridos nos mapas de isovalores de condutividade aparente. Estes indicativos sugerem que as áreas adjacentes ao poço de injeção de resíduos estão fase final de recuperação, com retorno ao seu estado natural. Este intervalo apresenta elevada cargabilidade, coincidência que sugere a neoformação de minerais em ambiente com maior oxigenação devido a concentrações bastante baixas de contaminantes, conforme sugere o modelo de degradação microbial de derivados de petróleo (Figura 1).

A presença de níveis lateríticos no perfil de solo indica que o Ferro é o elemento predominante na neoformação mineral. As diferenças entre os valores médios de cargabilidade e na quantidade relativa de oxigênio disponível, acima $36 \mathrm{~ms}$ na anomalia central da linha LG com predomínio de ambiente redutor e acima de $100 \mathrm{~ms}$ na a anomalia da linha LB com predomínio de ambiente oxidante, sugerem tipos minerais distintos.

É provável que haja neoformação de magnetita na área central da pluma de contaminantes. Devido à efetivação de processos de atenuação natural da fase contaminante e restituição das características naturais do ambiente, nas áreas adjacências deve haver neoformação de hematita, haja vista ser um processo que ocorre naturalmente na área não impactada, conforme indicam descrições em perfis de solo.

\section{Agradecimentos}

O presente trabalho foi realizado com o apoio do

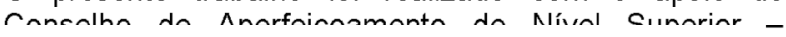
CAPES por meio da concessão de bolsa de estudos, a nartir dn nrnarama de Pós-aradılac:ãn em Gennciênncias, Instituto de Geociência e Ciências Exatas - IGCE, Universidade Estadual Paulista - UNESP.

\section{Referências}

Aquino, W. F., 2000. Métodos Geofísicos Eletromagnéticos aplicados ao diagnóstico da contaminação de Solo e das Águas Subterrâneas em área de Infiltração de Resíduos Industriais. $121 \mathrm{f}$. Dissertação (Mestrado em Geociências). Instituto de Geociências. Universidade de São Paulo.

Atekwana $F$ A Salırk W A Werkema .IR $\cap \Pi$., 2000. Investigations of geoelectrical signature at hydrocarbon contaminated site. Journal of Applied Geophysics, n44, p.167-180.

Benson, A. K., 1995. Applications of ground penetrating radar in assessing some geological hazards: examples of groundwater contamination, faults, cavities. Journal of Applied Geophysics, $n^{0} 33$, p. 177-193.

Benson, A. K., Payne, K. L., Stubben, M. A., 1997 Mapping groundwater contamination using dc resistivity and VLF geophysical methods - A case study. Geophysics, vol. 62 , no. 1, p. $80-86$.

Benson, A. K. \& Stubben, M. A., 1995. Interval resistivities and very low frequency electromagnetic induction-an aid to detecting groundwater contamination in space and time: A case study. Environmental Geosciencs, vol. 2, p. 74-84.

Bermejo, J. L., Sauck, W. A., Atekwana E. A.,1997. Geophysical discovery of a new LNAPL plume at the former Wurtsmith AFB. Ground Water Monitoring Remediation, vol. $17, n^{\circ} 4$, p. 131-137.

r.accidv $\cap$ P Werkema $\cap \cap$.lr Salı.k $W$ Atekwana, E., Rossbach, S., Duris, J., 2001. The Effects of LNAPL Biodegradation Products on Electrical Conductivity Measurements. Journal of Environmental \& Engineering Geophysics, vol. 6, no 1, p. 47-52.

Cetesb - Companhia de Tecnologia de Saneamento Ambiental, 1997. Relatório do levantamento geofísico para diagnóstico da contaminação da indústria Sulfabrás São Paulo: Cetesb, 91p

Ehrlich, H. L., 1996. How microbes influence mineral growth and dissolution. Chem. Geology, vol 132, p. $5-9$.

Fna - I Inited States Fnvirnnmental Protection Axenciv., 1998. Technical Protocol for evaluating natural attenuation of chlorinated solvents in ground water. Washington DC, epa/600/r-98/128

Fna - I Inited States Fnvirnnmental Protection Axenc:v., 1999. Microbial processes affecting monitored natural attenuation of contaminants in the subsurface. Ada, Oklahoma, epa/540/s-99/00.

Harter, R. D., 1977. Reactions of minerals with organic compounds in soil. Minerals in Soil, Environments Soil Science Society of America, p. 709-739.

Hiebert, F. K. \& Bennett, P. C., 1992. Microbial Control of Silicate Weathering in Organic-Rich Ground Water. Science: American Association for the Advancement of Science, vol. 258, p. $278-281$

Lima, O. A. L., Sato, H. K., Porsani, M. J., 1995. Imaging indıctrial rontaminant nlımes with recistivitu terhniaı Ies. Journal Applied Geophysics, vol. 34, p. 93-108.

Loke, M. H. \& Barker, R. D., 1996. Rapid least-squares inversion of apparent resistivity pseudosections by a duaci-Newton method Gennhvciral Procnentinn $\mathrm{n} 44 \mathrm{n}$. 131-152. 
Lovley, D. R., 1990. Magnetite formation during microbial dissimilatory iron reduction. In: R.B. Frankel and R. P. Blakemore (ed.), Iron biominerals, p. 151-166.

Mccabe, C., Sassen, R., Saffer, B., 1987. Occurrence of senondarv mannetite within hindearaded nil Genlnav vol. 15. p. $7-10$.
McMahon, P.B. \& Chapelle, F. H., 1991. Microbial production of organic acids in aquitard sediments and its

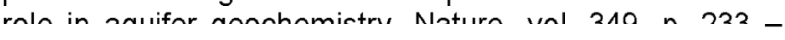
235

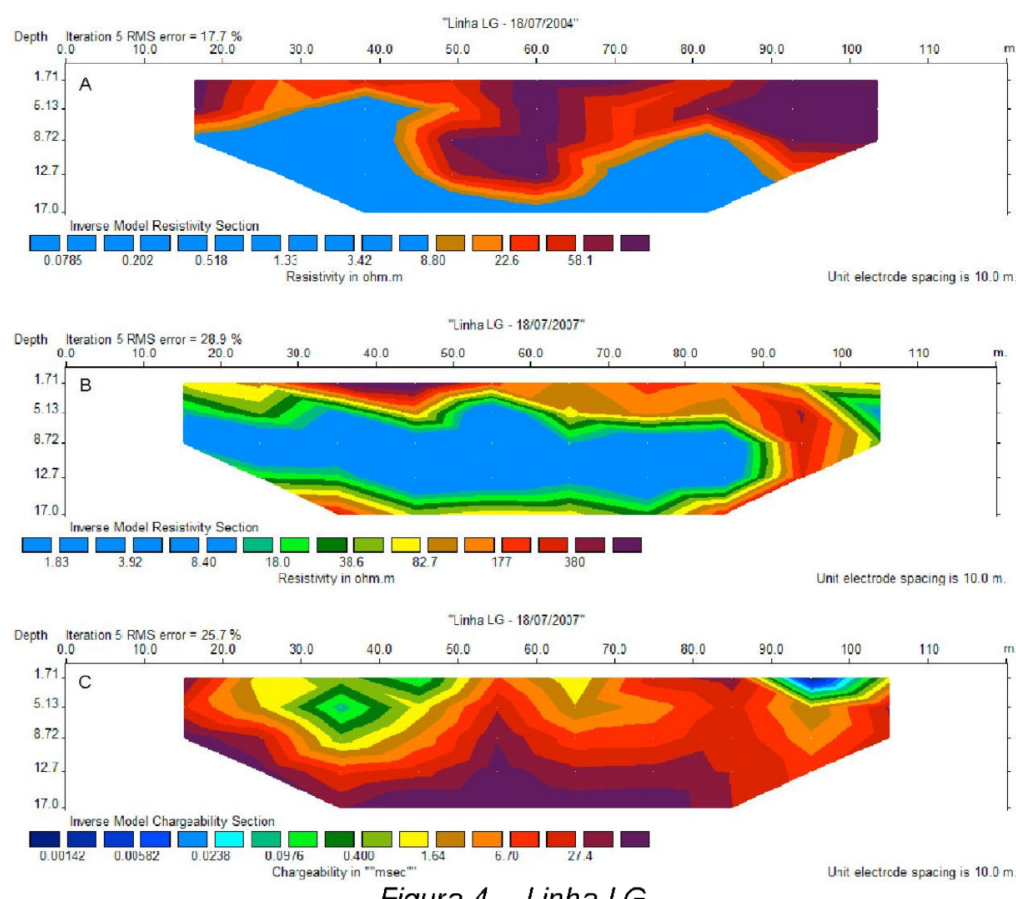

Figura 4 - Linha $L G$
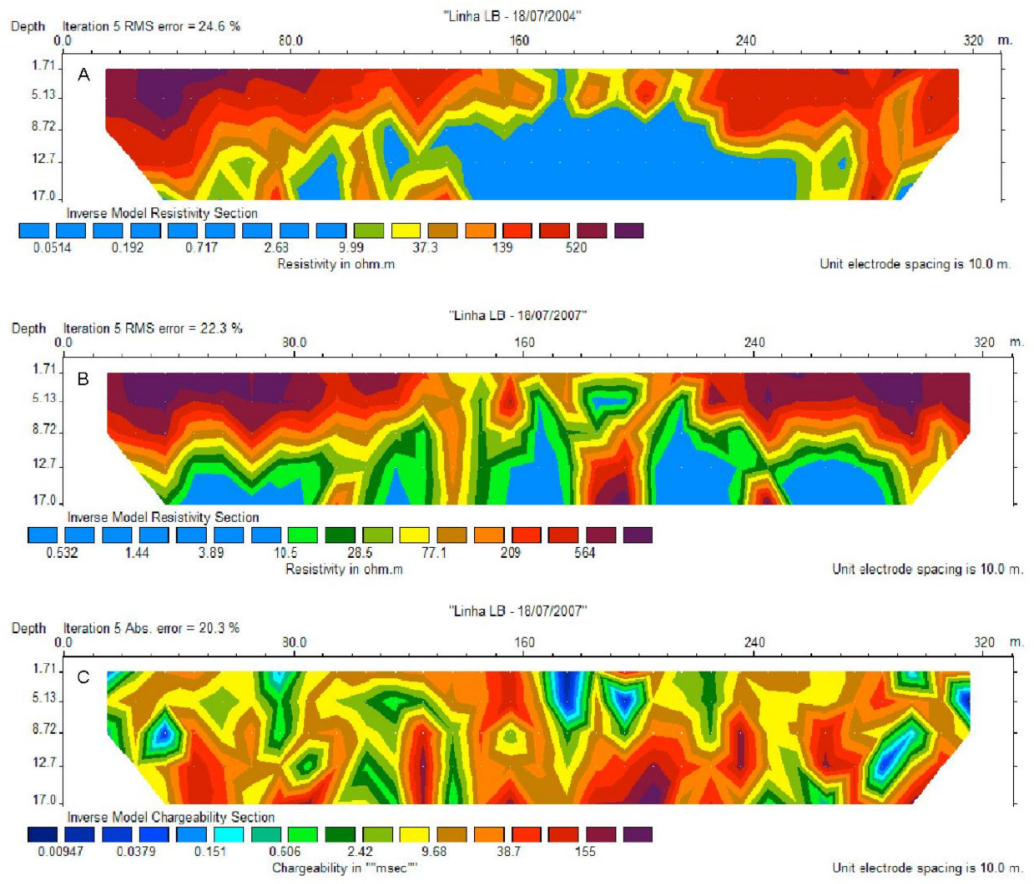

Figura 5 - Linha $L B$

III Simpósio Brasileiro da SBGf - Belém 2008 